Musées, Patrimoine et Culture scientifiques et techniques

$153 \mid 2014$

mai-juin 2014

\title{
Récolement et rénovation au muséum de Bordeaux
}

Nathalie Mémoire

\section{OpenEdition \\ Journals}

Édition électronique

URL : http://journals.openedition.org/ocim/1389

DOI : 10.4000/ocim.1389

ISSN : 2108-646X

Éditeur

OCIM

Édition imprimée

Date de publication : 25 juin 2014

ISSN : 0994-1908

Référence électronique

Nathalie Mémoire, "Récolement et rénovation au muséum de Bordeaux », La Lettre de l'OCIM [En ligne], 153 | 2014, mis en ligne le 26 juin 2016, consulté le 04 mai 2019. URL : http:// journals.openedition.org/ocim/1389; DOI : 10.4000/ocim.1389 


\section{Récolement et rénovation au muséum de Bordeaux}

\section{Nathalie Mémoire}

Conservateur en chef du Patrimoine,

directrice du muséum d'Histoire naturelle de Bordeaux
Un inventaire complet, documenté et illustré, avec un récolement achevé, serait l'outil idéal pour la préparation d'un projet de rénovation impliquant un nouveau parcours muséographique. Il permettrait d'effectuer efficacement la sélection des spécimens qui viendrait illustrer le synopsis, de prévoir leur restauration, de travailler de manière rationnelle à un programme d'acquisition, de fournir au scénographe le « catalogue de spécimens sélectionnés » qu’il attend, enfin de les préparer, parfaitement identifiés et localisés, en vue de leur installation dans le nouveau parcours.

Or, pour les rénovations engagées dans la décennie 2000 la difficulté est venue de la nécessité de concilier les obligations et les calendriers de deux projets, celui de la rénovation et celui du récolement. Dans certains cas, les chantiers de collections induits par les deux obligations profiteront l'un de l'autre, mais les différences de rythmes et d'objectifs ont de fait compliqué la tâche. Au moins six muséums ont ainsi engagé leur rénovation depuis 2000 : certaines sont achevées et les musées rouverts (La Rochelle, Toulouse, Bayonne), certains établissement se sont rénovés progressivement sans fermeture (Nantes), d'autres sont en cours (Lyon, Bordeaux).

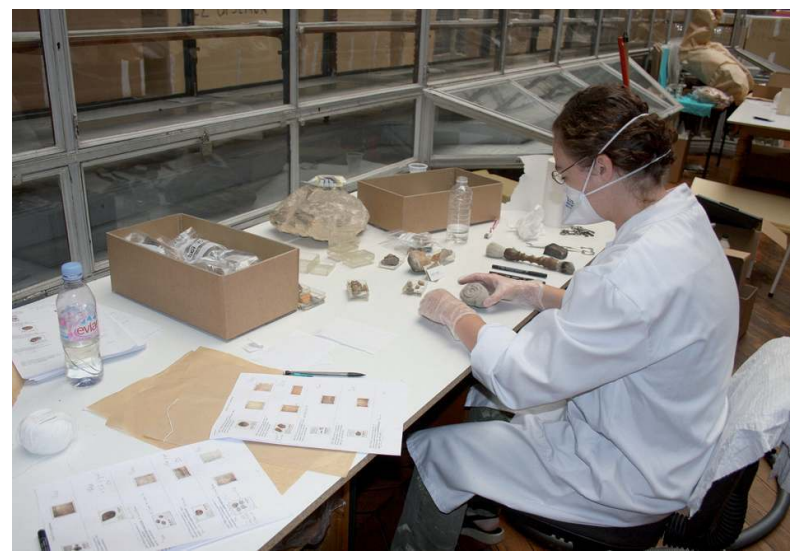

Lors du chantier des collections en vue du déménagement, les opérations de récolement, dépoussiérage, photographie, conditionnement, marquage des emballages individuels, des boîtes de conditionnement et des cartons de transfert sont menées de front.

(c) Muséum d'Histoire naturelle de Bordeaux/Hana Goodall
Enfin, le récolement des collections vise à vérifier la présence du bien, sa localisation et son état. Il apparaît aussitôt qu'un projet de rénovation, impliquant de vider tout ou partie d'un bâtiment de ses collections, de créer un nouveau parcours muséographique - et donc une nouvelle sélection des pièces à exposer-, puis une réinstallation de cette sélection, va encore compliquer considérablement les opérations de récolement du fait des multiples mouvements qui vont intervenir durant la période du récolement.

\section{Inventaire rétrospectif et localisation}

Au muséum d'Histoire naturelle de Bordeaux, aucun véritable inventaire au sens " Musée de France », ni même d'inventaire ancien, avec numérotation et marquage n'existait. Il a donc été décidé dès 1996 de réaliser des inventaires rétrospectifs face aux vitrines. La tâche était considérable, la fondation du musée remontant à 1791 et son installation dans les locaux actuels à 1862, l'aménagement des vitrines n'ayant quasiment pas varié depuis, dans l'esprit comme dans le mobilier.

Dès le début du pré-inventaire manuscrit en 1996, il est apparu indispensable de commencer par créer une typologie topographique permettant d'identifier précisément la localisation de chaque spécimen : pour chaque bâtiment furent définies une cartographie et une nomenclature détaillant l'étage, la vitrine dans l'étage, l'étagère, la position sur l'étagère. Lorsqu'en 2000, le programme d'informatisation, soutenu par le ministère de l'Enseignement supérieur et de la Recherche, a été engagé, seule la saisie des notices était envisagée. La révolution technique qu'a représenté l'avènement de la photographie numérique (avec son accessibilité en termes de coût comme de vitesse d'exécution) a permis d'engager, dès l'année 2002, des campagnes systématiques de photographie, couplées avec le travail de marquage afin de minimiser les manipulations. Ces deux actions (photographies et marquage) ont été ellesmêmes couplées avec un travail de conservation : dépoussiérage général et constat d'état, permettant d'alimenter et argumenter les choix en matière de sélection 
et restauration en vue du futur parcours muséographique, mais aussi d'enregistrer les données qui seraient nécessaires au récolement. Ainsi, dans un premier temps, nous avons décidé, avec l'accord des services de la DRAC, que l'inventaire rétrospectif vaudrait récolement.

\section{Projet de rénovation, chantier des collections et " grands mouvements"}

Car en parallèle, un projet de rénovation et extension de l'établissement était proposé à la Ville dès l'année 2000, suivi d'études de faisabilité et programmation, puis d'un arbitrage, d'un second projet et d'un programme validés fin 2006, enfin du choix de la maîtrise d'œuvre en $2007^{(1)}$. Le projet a pu être complété par celui de la construction d'un Centre de Conservation des Collections (CCC), pérenne, permettant de vider entièrement l'ancien musée et d'y transférer à terme les anciennes réserves.

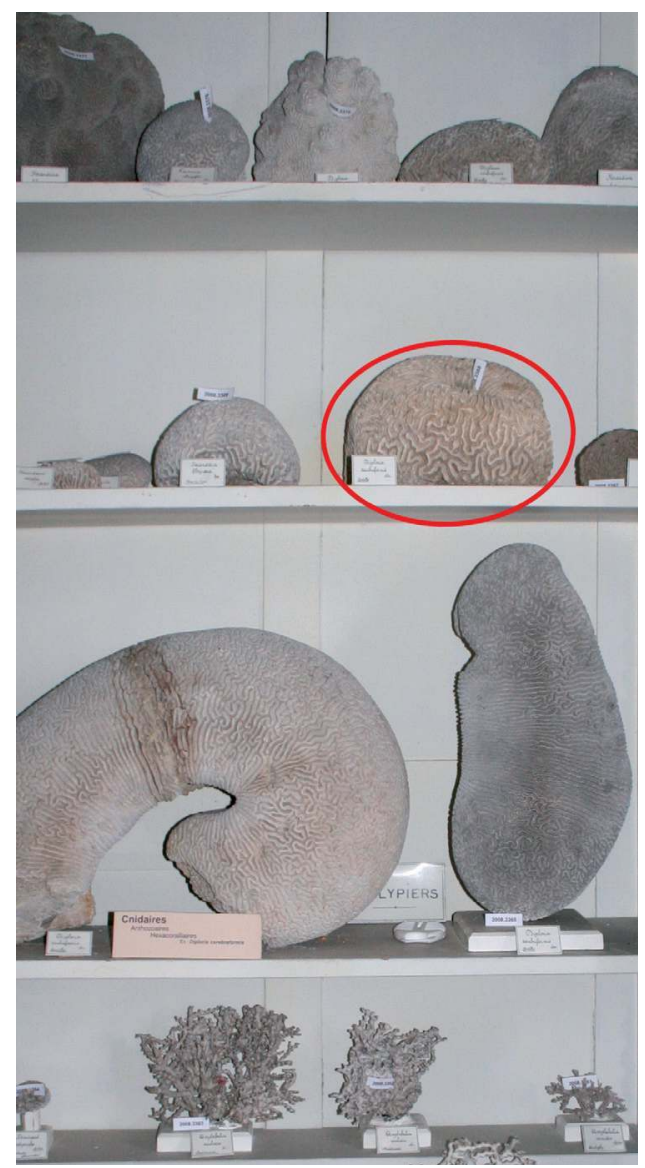

Afin de minimiser les manipulations, les opérations de marquage et photographie ont été différées et regroupées. Ainsi, lors des premières opérations d'inventaire rétrospectif face aux vitrines, les spécimens étaient identifiés par leur position dans la vitrine (ici cote 2.198.B.04, c'est-à-dire exposé au $2^{e}$ étage, vitrine $n^{\circ} 198$, tablette $\mathrm{B}-2 \mathrm{e}$ en partant du haut, $4^{\mathrm{e}}$ objet en partant de la gauche). Le spécimen était en outre cerclé sur la photographie de la vitrine et la photographie est insérée dans la notice informatisée. ○) Muséum d'Histoire naturelle de Bordeaux/Frédéric Deval
Ainsi, à partir de la fermeture du musée en 2009, un nouveau chantier des collections portant sur l'ensemble des collections, exposées ou non, a permis de compléter les opérations de marquage et photographie des collections difficilement accessibles (étagères situées entre $2 \mathrm{~m}$ et 3,50 $\mathrm{m}$ de hauteur), d'effectuer un dépoussiérage systématique de toutes les collections à transférer, d'enregistrer les constats d'état et de conditionner la totalité des collections en vue du déménagement, permettant de libérer les locaux pour le démarrage du chantier bâtiment. Il est alors apparu que la localisation ayant changé, l'inventaire rétrospectif ne pouvait plus seul valoir récolement...

Le plan de récolement présenté à la DRAC en juin 2011 a donc dû prendre en compte les mouvements imposés par le calendrier prévisionnel du chantier de rénovation (trois bâtiments concernés), soit un total de cinq mouvements majeurs entre le début du conditionnement des collections exposées (2009), les transferts vers le CCC, les retours en exposition une fois

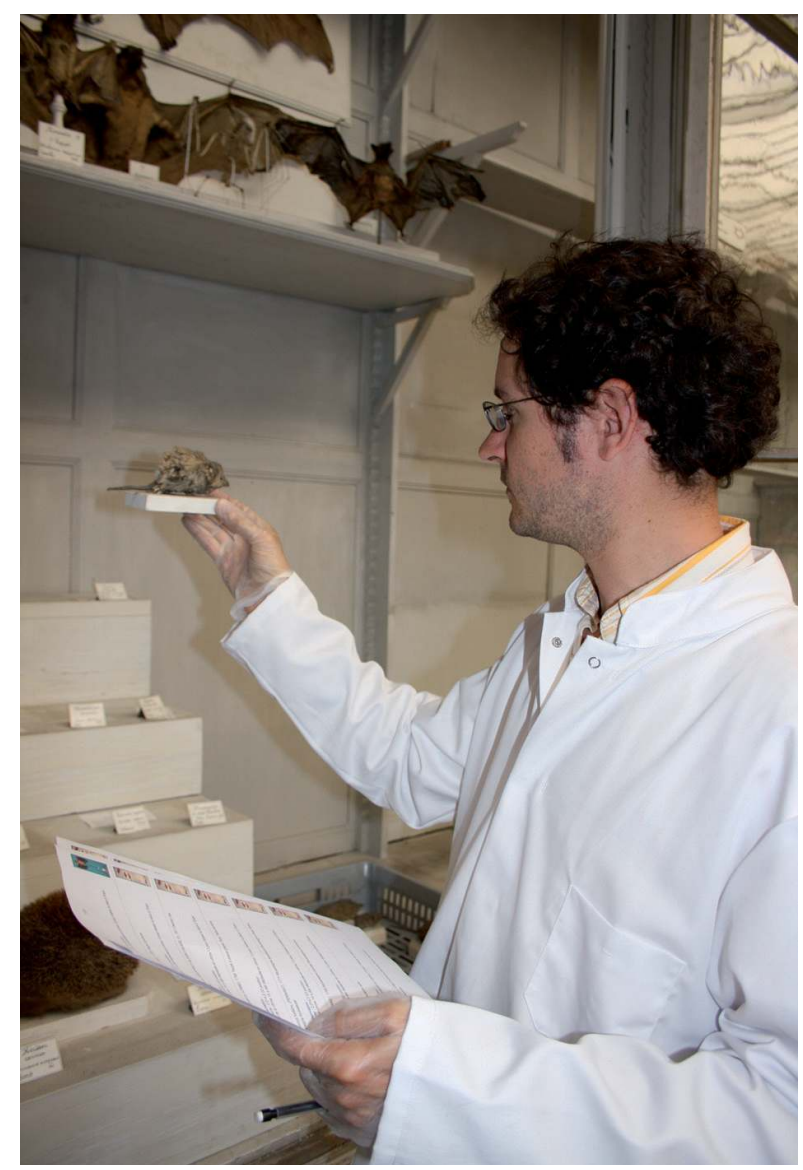

En 2009 et 2010 : le chantier des collections au muséum de Bordeaux, en vue du déménagement. Le récolement est mené à partir d'extraits imprimés de la base de données lorsque les vitrines sont vidées. (c) Muséum d'Histoire naturelle de Bordeaux/Hana Goodall 


\section{Points de vue complémentaires}

le musée rénové depuis le CCC ou les anciennes réserves, enfin le transfert final des collections restantes de l'ancienne réserve vers le CCC.

\section{Un retard mis à profit}

Cependant, le complexe chantier de réhabilitation du bâtiment principal (monument historique en centre ville, dans un jardin d'agrément) a été retardé. La réouverture qui était prévue fin 2014 est aujourd'hui différée d'au moins deux ans. Du point de vue du récolement, nous prenons ce retard comme un avantage. D'une part, les forces de l'équipe ont pu se concentrer majoritairement sur ce chantier et d'autre part, les mouvements induits par le réaménagement des salles pourront intervenir après la finalisation de ce premier récolement. Ce sont donc «seulement » trois mouvements qui seront intervenus et qu'il a fallu suivre pour la finalisation attendue en juin 2014. Mais faisons le pari que le travail ultérieur en sera facilité.

\section{Note}

(1) Groupement Basalt Architecture (mandataire), Die Werft Muséographie et Média, Coplan-Grontmij (bureau d'études techniques) et Impédance (acoustique).

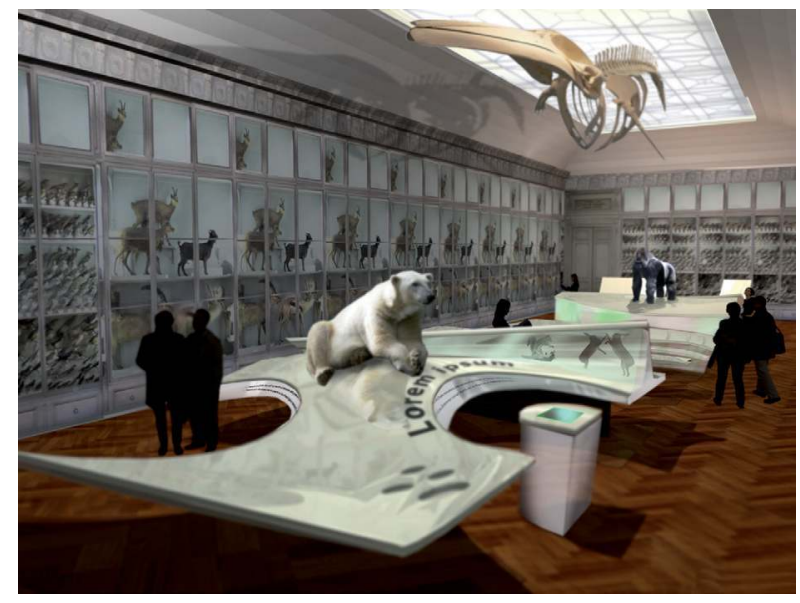

Projet de la rénovation-extension du muséum d'Histoire naturelle de Bordeaux. ๑ Basalt Architecture, Die Werft Muséographie et Média, Coplan, Impédance 